Short communication

\title{
Long-range connective sandpile models and its implication to seismicity evolution
}

\author{
Chien-chih Chen ${ }^{\mathrm{a}, *}$, Ling-Yun Chiao ${ }^{\mathrm{b}}$, Ya-Ting Lee ${ }^{\mathrm{a}}$, Hui-wen Cheng ${ }^{\mathrm{a}}$, Yih-Min $\mathrm{Wu}^{\mathrm{c}}$ \\ a Department of Earth Sciences and Graduate Institute of Geophysics, National Central University, Jhongli, Taiwan 320, ROC \\ ${ }^{\mathrm{b}}$ Institute of Oceanography, National Taiwan University, Taipei, Taiwan 106, ROC \\ c Department of Geosciences, National Taiwan University, Taipei, Taiwan 106, ROC
}

\section{A R T I C L E I N F O}

\section{Article history:}

Received 2 October 2007

Received in revised form 31 March 2008

Accepted 2 April 2008

Available online 10 April 2008

\section{Keywords:}

Self-organized criticality

Sandpile model

Long-range connection

Seismicity

b values

\begin{abstract}
A B S T R A C T
We propose a new variant of the sandpile model, the long-range connective sandpile model, by means of introducing randomly internal connections between two separated distant cells. The long-range connective sandpile model demonstrates various self-organized critical states with different scaling exponents in the power-law frequency-size distributions. We found that a sandpile with higher degree of randomly internal long-range connections is characterized by a higher value of the scaling exponent for the distribution, whereas the nearest neighbor sandpile is possessed of a lower scaling exponent. Our numerical experiments on the long-range connective sandpile models imply that higher degree of random long-range connections makes the earthquake fault system more relaxant that releases accumulated energy more easily and produces fewer catastrophic events, whereas lower degree of long-range connections possibly caused by fracture healing very likely motivates accelerating seismicity of moderate events.
\end{abstract}

(C) 2008 Elsevier B.V. All rights reserved.

\section{Introduction}

Many geophysical phenomena are scale invariant and exhibit the power-law distribution (Turcotte, 1997; Dodds and Rothman, 2000), which is the only statistical distribution not including a characteristic scale. A striking example is the Gutenberg-Richter relation for the frequency-magnitude statistics of earthquakes. The scaling exponent and its associated variation is then a matter of fundamental importance in such power-law distribution. Specifically, in the study of seismicity evolution, the scaling exponent in the Gutenberg-Richter relation, which is well known as the $b$-value, has been very often discussed in the literature and considered as a monitoring index related to the forthcoming large earthquakes (Smith, 1986; Urbancic et al., 1992; Wiemer and Wyss, 1994; Henderson et al., 1994; Guo and Ogata, 1995; Legrand et al., 1996; Wyss, 1997; Lapenna et al., 1998; Henderson et al., 1999; Barton et al., 1999; Oncel and Wilson, 2004; Wyss et al., 2004; Mandal et al., 2005; Wu and Chiao, 2006). The reductions in the $b$-value before a large earthquake have been reported in many researches. The reduced $b$-value is probably caused by the quiescence of smaller earthquakes and/or the activation of moderate earthquakes (e.g. Chen, 2003; Chen et al., 2005; Wu and Chiao, 2006). For example, observed before the $1999 M_{\mathrm{w}} 7.6$ Chi-Chi,

\footnotetext{
* Corresponding author. Institute of Geophysics, National Central University, Jhongli, Taiwan 320, ROC. Tel.: +88634227151 65653; fax: +88634222044.

E-mail address: chencc@ncu.edu.tw (C. Chen).
}

Taiwan earthquake were the quiescence of earthquakes with magnitudes smaller than 4 (Fig. 4 in Wu and Chiao, 2006) and activation of events with magnitudes larger than 5 (Fig. 3 in Chen, 2003).

Numerical experiments in tending to comprehend seismicity had mainly been based on simple conceptual models such as the springslider model of Burridge and Knopoff (1967), the sandpile model of Bak et al. (1987), the block structure model of Gabrielov et al. (1990), and the lattice-solid model of Mora and Place (1994). Among them two types of simple cellular automata models are the spring-slider model (Burridge and Knopoff, 1967) and the sandpile model (Bak et al., 1987). In the sandpile model a hallmarked state, which is very well known as the self-organized criticality (SOC) state and characterized by the frequency-size power-law distribution, is established solely because of the dynamical interactions among individual elements of the system. Since the concept of self-organized criticality was introduced in Bak et al. (1987), earthquakes have been identified as an example of this phenomenon in nature (Bak and Tang, 1989; Sornette and Sornette, 1989; Ito and Matsuzaki, 1990) and the observation of the Gutenberg-Richter law has been suggested to be the manifestation of the self-organized critical state of the dynamics of the earthquake faults.

For earthquake studies, the sandpile model sheds new insights into the earthquake physics in addition to those derived from earlier, much complicated spring-slider models (Burridge and Knopoff, 1967; Rundle and Jackson, 1977; Carlson et al., 1994). Here we propose to 
invoke an alternative variant of the sandpile model to demonstrate variations in the scaling exponent of the power-law distribution by means of introducing randomly internal connections between separated distant cells. Note that the internal networks of either the conventional spring-slider or sandpile models are essentially the nearest neighboring networks. We examine the systematic impacts on the scaling exponents of these randomly distant connections in our modified sandpile model. The implications on the earthquake fault systems are then discussed in the end of this paper.

\section{Long-range connective sandpile models and its frequency-size distributions}

We build our sandpile model by a very simple set of rules that is very similar to the original one (Bak et al., 1987). For a square grid of $L$ by $L$ cells, we randomly throw sands, one at a time, onto the grid. In the original sandpile model, once the total amount of the accumulated sands within a single cell reaches the threshold amount of 4 , they will be redistributed to the four adjacent cells (the nearest neighbors) or lost off the edge of the grid. All the cells receiving grains from their neighbors will be checked, and the redistribution would be continued further away if any one of them reaches consequently over the threshold too. For each throw of new grains, the redistribution proceeds until none of the meshes receiving new grains exceeds the threshold. The total amount of cells involved in the redistribution process initiated by a single throw is defined to be the size of the event. Note that the total amount of grains retained within the grid increases linearly in the beginning transient thousands of iterations and then stays at a quasi-static value with small fluctuations.

Our modified rule of randomly internal connections is very similar to the implementation of Watts and Strogatz (1998). For any particular cell, when the accumulated grains exceed the threshold and redistribution occurs, one of the original nearest neighbor connections confronts a chance with prescribed connective probability $P_{\mathrm{c}}, 0<P_{\mathrm{c}}<1$, of redirecting to a randomly chosen, distant cell and so the original connection is replaced by a randomly chosen mesh that might be faraway from the toppling cell. We may call such version of sandpile model the long-range connective sandpile (LRCS) model. Apparently, when $P_{\mathrm{c}}=0$, the LRCS model reduced to the original nearest neighbor sandpile model.

We perform a series of simulations each with $10^{6}$ throws of single grain on a square grid with $50 \times 50$ meshes, starting with the original nearest neighbor sandpile $\left(P_{\mathrm{c}}=0\right)$ and then gradually increasing the connective probability $P_{\mathrm{c}}$. For each $P_{\mathrm{c}}$, the frequency-size power law and the manifested SOC state always emerge robustly. The power-law frequency-size distributions demonstrating the scale invariance on the $\log -\log$ plot for two experiments with $P_{\mathrm{c}}=0$ and $P_{\mathrm{c}}=1$ are very distinct (Fig. 1). The breakdown of the power law at the extreme of great events is obviously due to the limited size of the experimental grid, the so-called finite-size effect.

The frequency-size power law is not a linear-normal relation. Hence, all the data points for the distribution need not to have the same weight as supposed when a linear relation is regressed with a standard least-square method. For example, if the distribution has two large events instead of one, it will change very much the result from using a least-square method. To address the issue of different weighting in a power-law distribution, we adopt a weighted leastsquare method to assign different fitting weights for different data points of the distribution (Shi and Bolt, 1982). The results lead to a scaling exponent of 1.06 characterizing the frequency-size statistics for the original nearest neighbor sandpile model (Bak et al., 1987), whereas a higher scaling exponent of 1.32 for the LRCS model with $P_{\mathrm{C}}=1$ (Fig. 1).

As gradually increasing the connective probability $P_{\mathrm{c}}$ from 0 to 1 , we have found that there is a systematic steady increase of the scaling exponent along with a notable decrease of the amount of total grains staying on the grid (Fig. 2). The comparison suggests that, with higher degree of random long-range connections that is obtained by invoking higher connective probability $P_{\mathrm{c}}$, the critical state is characterized by a sandpile with more small avalanches involving only a few cells and, consequently, the higher value of the scaling exponent for the frequency-size distribution. In other words, higher degree of random long-range connections actually makes the system more relaxant that releases the potential of building up catastrophic avalanches more easily and produces fewer catastrophic avalanches.

\section{Conclusion and discussion}

There have been reported that an observed feature associated with the earthquake statistics is a significant increase of moderate earthquakes and a noticeable reduction of the $b$-value of the GutenbergRichter relationship prior to great earthquakes (e.g. Sykes and Jaume, 1990; Henderson et al., 1994; Bowman et al., 1998; Jaume and Sykes, 1999; Chen, 2003; Wu and Chiao, 2006). An immediate implication of our experiments to the temporal evolution of the seismicity is that, as the tectonic loading is slowly driving the earthquake fault system through the cycle of earthquakes leading to the catastrophic event, there might be different phases all bearing characteristics of SOC. The initial phase is characterized by a small but notable random longrange connection with $1>P_{\mathrm{c}} \gg 0$. It then evolves gradually towards more and more regular nearest neighbor connection with $P_{\mathrm{c}} \sim 0$ such that the manifestation is the noted accelerating seismicity of moderate size and a reduction of the $b$-value (Fig. 2). After the occurrence of some great earthquakes, possibly due to the activity of faults, the change of pore water pressure or the dynamical triggering of seismic waves, the earthquake fault system is very likely to bear with a significant degree of long-range connections with higher $P_{\mathrm{c}}$. Bursting aftershock activity might well be the manifestation of a raised longrange connection. Then, the system heals gradually along with the tectonic loading and gets back to the initial phase preparing for the next cycle of extreme events.

The mechanism depicted above is utterly different from the temporal growth of long-range correlation length on the system in the critical point theory of earthquakes (Bowman et al., 1998; Jaume and Sykes, 1999; Rundle et al., 1999; Rundle et al., 2000). To the critical point theory of earthquakes, the correlation length of the regional stress field grows prior to the catastrophic event. The origin of accelerating seismicity of moderate events lies in the growing

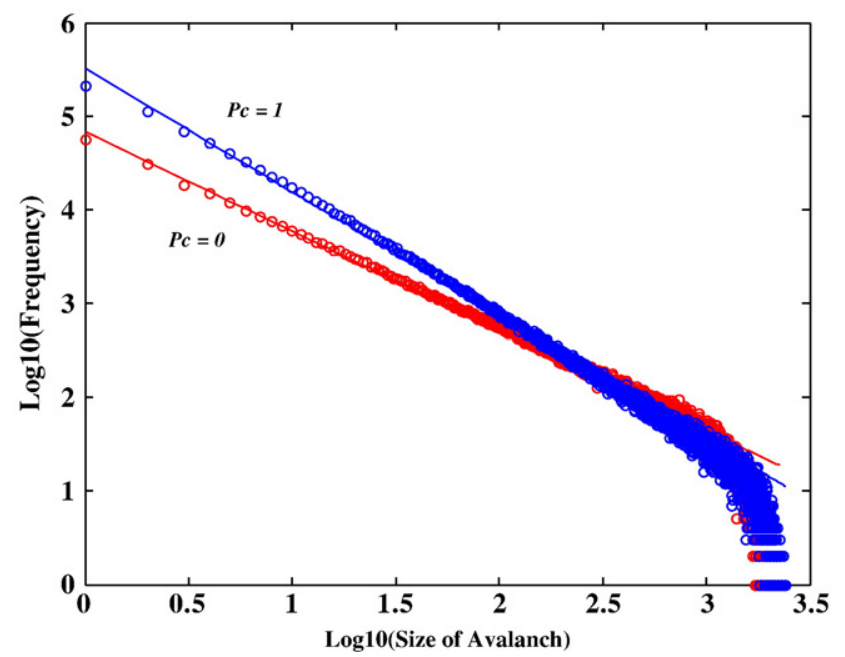

Fig. 1. Power-law frequency-size distributions for two simulations of the long-range connective sandpile models with $P_{\mathrm{c}}=0$ and $P_{\mathrm{c}}=1$, respectively. Also shown are two regression lines, with slopes of 1.06 and 1.32 , best fitting the power-law distributions from the weighted least-square method. Note that the long-range connective sandpile with $P_{\mathrm{c}}=0$ is identical to the original nearest neighbor sandpile by Bak et al. (1987). 


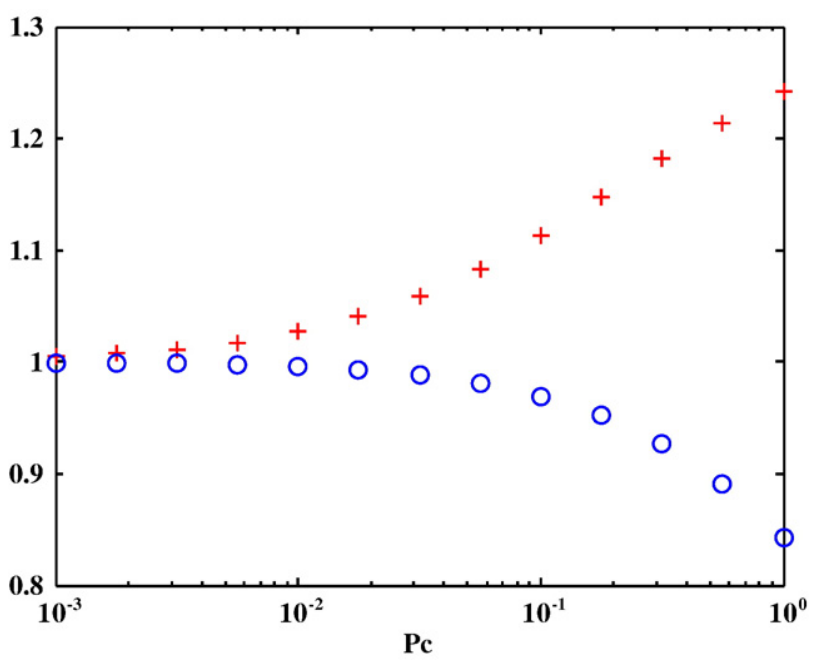

Fig. 2. Scaling exponents (crosses) of the long-range connective sandpile models with various connective probabilities $P_{\mathrm{c}}$ gradually increasing from 0 to 1 . All the scaling exponents are normalized to the value of scaling exponent, i.e. 1.06, of the nearest neighbor sandpile with $P_{\mathrm{c}}=0$. Also shown in the plot is the average number of total grains staying on the grid (circles) for each $P_{\mathrm{c}}$. Again, the total grain number is normalized to the number for the case of $P_{\mathrm{c}}=0$, which is averagely 5244 .

correlation length which sets a limit on the largest possible magnitude of the forthcoming catastrophic event. In other words, the occurrence of catastrophic event strongly relies on the power-law growth of the correlation length (Rundle et al., 1999) and the power-law increase in the cumulative Benioff strains (Bowman et al., 1998). In the LRCS model high degree of long-range connections/interactions does not necessarily encourage catastrophic earthquakes and, perhaps even more interestingly, it is lower degree of long-range connections/ interactions that motivates accelerating seismicity of moderate events. It is the case without any long-range connections $\left(P_{\mathrm{c}}=0\right)$ such that the interactions are limited within the nearest neighbors that yields the lowest scaling exponent and the most enriched events above moderate sizes. It is probably that the long-range correlation and the long-range connection are two different concepts regarding the spatial scale. In other words, reducing global long-range connection actually helps to build regional long-range correlation of stress field.

It is also interesting to compare the results from the LRCS models with the spring-slider models (e.g. Shaw et al., 1992; Wang et al., 1995; Wang, 1997). Many researchers have tried to investigate the seismic activation preceding a large earthquake with the spring-slider model. Shaw et al. (1992), for example, found that the seismic activity accelerates dramatically prior to a large event in their spring-slider model and is usually a maximum in the neighborhood of the future epicenter. Numerical simulations in Wang et al. (1995) and Wang (1997) clearly demonstrated the distributions with different scaling exponents in the frequency versus rupture length or magnitude of earthquakes for various stiffness ratios in the spring-slider models. The stiffness ratio indicates the degree of the joint effect of the coil springs $K_{\mathrm{c}}$ between mass elements and the leaf springs $K_{\mathrm{l}}$ between the moving plate and the mass elements, and is defined as $K_{\mathrm{c}} / K_{\mathrm{l}}$. In the case of smaller stiffness ratio the frequency-magnitude distribution of earthquakes possesses a higher and steep slope in the log-log plot while larger stiffness ratio a lower and gentle slope for the distribution. Note that, when $K_{1}$ is much smaller than $K_{\mathrm{c}}$ or approaches to zero, the coupling effect due to the leaf spring disappears and the behavior of the system is dominated by the nearest neighbor coupling between two mass elements. For the case not including the frictional action, the spring-slider system with an infinite stiffness ratio is exactly reduced to a conservatively nearest neighbor sandpile model without long-range connections. As $K_{1}$ increases and the stiffness ratio decreases the coupling of the leaf springs takes away the accumulated energy of the system of mass elements and the energy redistribution process is no more localized to neighboring mass elements. We thus speculate that the effect of introducing a large $K_{1}$ is equivalent to that introducing a certain degree of long-range connections of distant mass elements. Our speculation is supported by the frequency-magnitude distributions with larger scaling exponents of synthetic seismicity for smaller stiffness ratios in the studies of Wang et al. (1995) and Wang (1997).

Very recently, there are some researches (e.g. Weatherley et al., 2002; Xia et al., 2005) demonstrating another type of the so-called long-range effect in the automaton or spring-slider models. Weatherley et al. (2002) used a long-range strain redistribution rule in their automaton model to approximate the elastodynamic stress Green's function of the crust. Similarly, Xia et al. (2005) presented the simulation of the springslider model with the long-range stress transfer. Note that, precisely speaking, those models actually employed a larger number of neighboring cells surrounding the failed cell for the longer-range transfer of stress/strain, which is essentially different from the redirection to a randomly chosen, distant cell in our LRCS models.

\section{Acknowledgements}

CCC is grateful for research support from both the National Science Council (ROC) and the Institute of Geophysics (NCU, ROC). LYC is supported by the National Science Council of the Republic of China under grant NSC 96-2611-M-002-001. Thanks are also extended to one anonymous reviewer for improving the manuscript.

\section{References}

Bak, P., Tang, C., 1989. Earthquakes as a self-organized critical phenomenon. J. Geophys. Res. 94, 15635-15637.

Bak, P., Tang, C., Wiesenfeld, K., 1987. Self-organized criticality: an explanation of $1 / \mathrm{f}$ noise. Phys. Rev. Lett. 59, 381-384.

Barton, D.J., Foulger, G.R., Henderson, J.R., Julian, B.R., 1999. Frequency-magnitude statistics and spatial correlation dimensions of earthquakes at Long Valley caldera, California,. Geophys. J. Int. 138, 563-570.

Bowman, D.D., Ouillon, G., Sammis, G., Sornette, A., Sornette, D., 1998. An observational test of the critical earthquake concept. J. Geophys. Res. 103, 24359-24372.

Burridge, R., Knopoff, L., 1967. Model and theoretical seismicity. Bull. Seismol. Soc. Am. 57, 341-371.

Carlson, J.M., Langer, J.S., Shaw, B.E., 1994. Dynamics of earthquake faults. Rev. Mod. Phys. 66, 657-670

Chen, C.C., 2003. Accelerating seismicity of moderate-sized earthquakes before the 1999 Chi-Chi, Taiwan, earthquake: testing time-prediction of self-organizing spinodal model of earthquakes. Geophys. J Int. 155, F1-F5.

Chen, C.C., Rundle, J.B., Holliday, J.R., Nanjo, K.Z., Turcotte, D.L., Li, S.C., Tiampo, K.F., 2005. The 1999 Chi-Chi, Taiwan, earthquake as a typical example of seismic activation and quiescence. Geophys. Res. Lett. 32 (22), L22315. doi:10.1029/2005GL023991.

Dodds, P.S., Rothman, D.H., 2000. Scaling, universality, and geomorphology. Annu. Rev. Earth Planet. Sci. 571-610.

Gabrielov, A.M., Levshina, T.A., Rotwain, I.M., 1990. Block model of earthquake sequence. Phys. Earth. Planet. Inter. 61, 18-28.

Guo, Z., Ogata, Y., 1995. Correlation between characteristic parameters of aftershock distributions in time, space, and magnitude. Geophys. Res. Lett. 22, 993-996.

Henderson, J., Main, I.G., Pearce, R.G., Takeya, M., 1994. Seismicity in north-eastern Brazil - fractal clustering and the evolution of the $b$-value. Geophys. J. Int. 116, 217-226.

Henderson, J.R., Barton, D.J., Foulger, G.R., 1999. Fractal clustering of induced seismicity in the Geysers geothermal area, California. Geophys. J. Int. 139, 317-324.

Ito, K., Matsuzaki, M., 1990. Earthquakes as self-organized critical phenomena. J. Geophys. Res. 95, 6853-6860.

Jaume, S.C., Sykes, L.R., 1999. Evolving towards a critical point: a review of accelerating seismic moment/energy release prior to large and great earthquakes. Pure Appl. Geophys. 155, 279-306.

Lapenna, V., Macchiato, M., Telesca, L., 1998. $1 / f^{b}$ fluctuations and self-similarity in earthquake dynamics - observational evidences in southern Italy. Phys. Earth Planet. Inter. 106, 115-127.

Legrand, D., Cisternas, A., Dorbath, L., 1996. Multifractal analysis of the 1992 Erzincan aftershock sequence. Geophys. Res. Lett. 23 (9), 933-936.

Mandal, P., Mabawonku, A.O., Dimri, V.P., 2005. Self-organized fractal seismicity of reservoir triggered earthquakes in the Koyna-Warna seismic zone, western India. Pure Appl. Geophys. 162, 73-90.

Mora, P., Place, D., 1994. Simulation of the frictional stick-slip instability. Pure Appl. Geophys. 143, 61-87.

Oncel, A.O., Wilson, T., 2004. Correlation of seismotectonic variables and GPS strain measurements in western Turkey. J. Geophys. Res. 109 (B11), B11306. 
Rundle, J.B., Jackson, D.D., 1977. Numerical simulation of earthquake sequences. Bull. Seismol. Soc. Am. 67, 1363-1377.

Rundle, J.B., Klein, W., Gross, S., 1999. Physical basis for statistical patterns in complex earthquake populations: models, predictions and tests. Pure Appl. Geophys. 155 575-607.

Rundle, J.B., Klein, W., Turcotte, D.L., Malamud, B.D., 2000. Precursory seismic activation and critical-point phenomena. Pure Appl. Geophys. 157, 2165-2182.

Shaw, B.E., Carlson, J.M., Langer, J.S., 1992. Patterns of seismic activity preceding large earthquakes. J. Geophys. Res. 97, 479-488.

Shi, Y., Bolt, B.A., 1982. The standard error of the magnitude-frequency $b$ value. Bull. Seismol. Soc. Am. 72, 1677-1687.

Smith, W.D., 1986. Evidence of precursory changes in the frequency-magnitude $b$ value. Geophys. J. R. Astron. Soc. 86, 815-838.

Sornette, A., Sornette, D., 1989. Self-organized criticality and earthquakes. Europhys. Lett. 9 197-202.

Sykes, L.R., Jaume, S.C., 1990. Seismic activity on neighboring faults as a long-term precursor to large earthquakes in the San Francisco Bay area. Nature 348, 595-599.

Turcotte, D.L., 1997. Fractals and Chaos in Geology and Geophysics, 2nd Ed. Cambridge Univ. Press, Cambridge.

Urbancic, T.I., Trifu, C.I., Long, J.M., Young, R.P., 1992. Space-time correlations of $b$ values with stress release. Pure Appl. Geophys. 139, 449-462.

Wang, J.H., 1997. On the frequency distribution of rupture lengths of earthquakes synthesized from a one-dimensional dynamical lattice model. J. Phys. Earth 45, 363-381.
Wang, J.H., Matsuzaki, M., Koyama, J., 1995. Dynamical modeling of earthquake occurrences. In: Koyama, J., Feng, D. (Eds.), Advance in Mathematical Seismology. Seismological Press, Beijing, pp. 148-164.

Watts, D.J., Strogatz, S.H., 1998. Collective dynamics of “small-world” networks. Nature 393, 440-442.

Weatherley, D., Mora, P., Xia, M.F., 2002. Long-range automaton models of earthquakes: power-law accelerations, correlation evolution, and mode-switching. Pure Appl. Geophys. 159, 2469-2490.

Wiemer, S., Wyss, M., 1994. Seismic quiescence before the landers $(M=7.5)$ and big bear $(M=6.5) 1992$ earthquakes. Bull. Seismol. Soc. Am. 84, 900-916.

Wu, Y.M., Chiao, L.Y., 2006. Seismic quiescence before the 1999 Chi-Chi, Taiwan Mw7.6 earthquake. Bull. Seismol. Soc. Am. 96, 321-327.

Wyss, M., 1997. Nomination of precursory seismic quiescence as a significant precursor. Pure Appl. Geophys. 149, 79-114.

Wyss, M., Sammis, C.G., Nadeau, R.M., Wiemer, S., 2004. Fractal dimension and $b$-value on creeping and locked patches of the San-Andreas fault near Parkfield, California. Bull. Seismol. Soc. Am. 94, 410-421.

Xia, J., Gould, H., Klein, W., Rundle, J.B., 2005. Simulation of the Burridge-Knopoff model of earthquakes with variable range stress transfer. Phys. Rev. Lett. 95, 248501. 